\title{
Nearly Space-Filling Fractal Networks of Carbon Nanopores
}

\author{
P. Pfeifer, ${ }^{1,2}$ F. Ehrburger-Dolle, ${ }^{3, *}$ T.P. Rieker, ${ }^{4, \dagger}$ M. T. González, ${ }^{5}$ W. P. Hoffman, ${ }^{6}$ M. Molina-Sabio, ${ }^{5}$ \\ F. Rodríguez-Reinoso, ${ }^{5}$ P. W. Schmidt,,${ }^{1, \sharp}$ and D. J. Voss ${ }^{1}$ \\ ${ }^{1}$ Department of Physics, University of Missouri, Columbia, Missouri 65211 \\ ${ }^{2}$ Center for Nonlinear Studies, Los Alamos National Laboratory, Los Alamos, New Mexico 87545 \\ ${ }^{3}$ Institut de Chimie des Surfaces et Interfaces, CNRS, F-68057 Mulhouse, France \\ ${ }^{4}$ Center for Microengineered Materials, University of New Mexico, Albuquerque, New Mexico 87131 \\ ${ }^{5}$ Departamento de Química Inorgánica, Universidad de Alicante, E-03080 Alicante, Spain \\ ${ }^{6}$ Air Force Research Laboratory, Edwards Air Force Base, California 93524
}

(Received 11 January 2001; revised manuscript received 17 October 2001; published 28 February 2002)

Small-angle x-ray scattering, nitrogen adsorption, and scanning tunneling microscopy show that a series of activated carbons host an extended fractal network of channels with dimension $D_{p}=2.8-3.0$ (pore fractal), channel width 15-20 $\AA$ (lower end of scaling), network diameter 3000-3400 $\AA$ (upper end of scaling), and porosity of $0.3-0.6$. We interpret the network as a stack of quasiplanar invasion percolation clusters, formed by oxidative removal of walls between closed voids of diameter of $\sim 10 \AA$ and held in registry by fibrils of the biological precursor, and point out unique applications.

DOI: $10.1103 /$ PhysRevLett.88.115502

Since the first experimental studies of fractal surfaces of disordered solids [1], it has been conjectured that situations may exist in which the pore space - as opposed to the solid, or the surface alone-is fractal. Such fractal networks of channels crisscrossing the solid, termed pore fractals or "negative image" of mass fractals, have attracted interest as a laboratory for unusual dynamics of confined processes, induced by the long-range correlation of the pore space. The scaling laws predicted relate the dynamic exponents to the fractal and spectral dimensions (spacefilling and branching properties) of the network and include anomalous diffusion, reaction, free motion [2], phase transitions [3], electric conduction of pore fluid [4], and hydrodynamic flow [5]. Here we report the first welldocumented case of a pore fractal.

The network is a new member in the family of nanostructured carbons. Its channel width is $15-20 \AA$, comparable to the width of single-wall carbon nanotubes, but instead of forming an assembly of freestanding tubes or bundle of tubes, the channels are embedded in a solid and connected. The network is of multiple interest: (i) Its synthesis differs vastly from that of isolated nanotubes. Created by controlled oxidation, a mainstay of mass production of porous carbons, it promises to be a low-cost competitor of isolated nanotubes for gas storage. (ii) For gas storage, it has outstanding mechanical stability, nanofluidic properties (rapid transport through branched channels), and capacity (high porosity, condensation in high dimensions) compared to nanotube bundles [6]. (iii) It offers a stage for "chemistry in confined spaces" and control of pathways similar to zeolites and other microscopic vessels [7]. (iv) The extended scaling regime, created by what we believe is invasion percolation, provides a unique platform to compare predicted dynamic exponents with experiment.
PACS numbers: 61.10.Eq, 47.55.Mh, 61.43.Hv, 81.05.Rm

Sandstones [4] and Vycor glass [8] have been put forth as pore fractals, but these proposals have been controversial or withdrawn [9]. Pore fractals are more challenging to ascertain than mass or surface fractals because they do not reveal their fractality when probed with material yardsticks: the pore-size distribution of a pore fractal is a delta function (mass and surface fractals give a power law), so an intruding nonwetting liquid, capillary condensate, or adsorbed layer will either fill the entire pore space or leave it entirely empty, depending on whether the radius of curvature of the liquid, or adsorbate size, is smaller or larger than the channel. Such an experiment shows the presence of channels, but gives no information about their spatial arrangement.

The porous carbons in this paper are activated carbons [10-13], prepared as follows [12]. Olive stones were carbonized in $\mathrm{N}_{2}$ at $850{ }^{\circ} \mathrm{C}$, yielding a char denoted by $\mathrm{H} 0$. The char was oxidized by $\mathrm{H}_{2} \mathrm{O}$ at $750{ }^{\circ} \mathrm{C}\left(\mathrm{C}+\mathrm{H}_{2} \mathrm{O} \rightarrow\right.$ $\mathrm{CO}+\mathrm{H}_{2}$ ) at $1 \%$ carbon per hour, yielding carbons with mass loss of $8 \%-74 \%$, denoted by $\mathrm{H} 8-\mathrm{H} 74$. The surface geometry of the samples (ground, dried) was investigated by small-angle $x$-ray scattering (SAXS). The scattered intensity at momentum transfer $q, I(q)$, was measured on a Bonse-Hart spectrometer for $q<0.1 \AA^{-1}$ and a pinhole spectrometer for $q>0.01 \AA^{-1}$ [14]. This gives data over an unusually wide range, $0.0003 \leq q \leq 0.7 \AA^{-1}$, with one decade of overlap of the two instruments.

To understand the scattering curves and their evolution with progressive oxidation (Fig. 1), we need to consider all three types of fractals. The intensity from a surface/mass/pore fractal obeys the power law $I(q) \propto$ $q^{D_{\mathrm{s}}-2\left(D_{\mathrm{m}}+D_{\mathrm{p}}\right)+6}[15,16]$, where $D_{\mathrm{s}}, D_{\mathrm{m}}$, and $D_{\mathrm{p}}$ is the fractal dimension of the surface, mass, and pore space, respectively. For a surface fractal, $D_{\mathrm{m}}=D_{\mathrm{p}}=3$, it reduces to the Bale-Schmidt scattering law, $I(q) \propto q^{D_{\mathrm{s}}-6}$ 


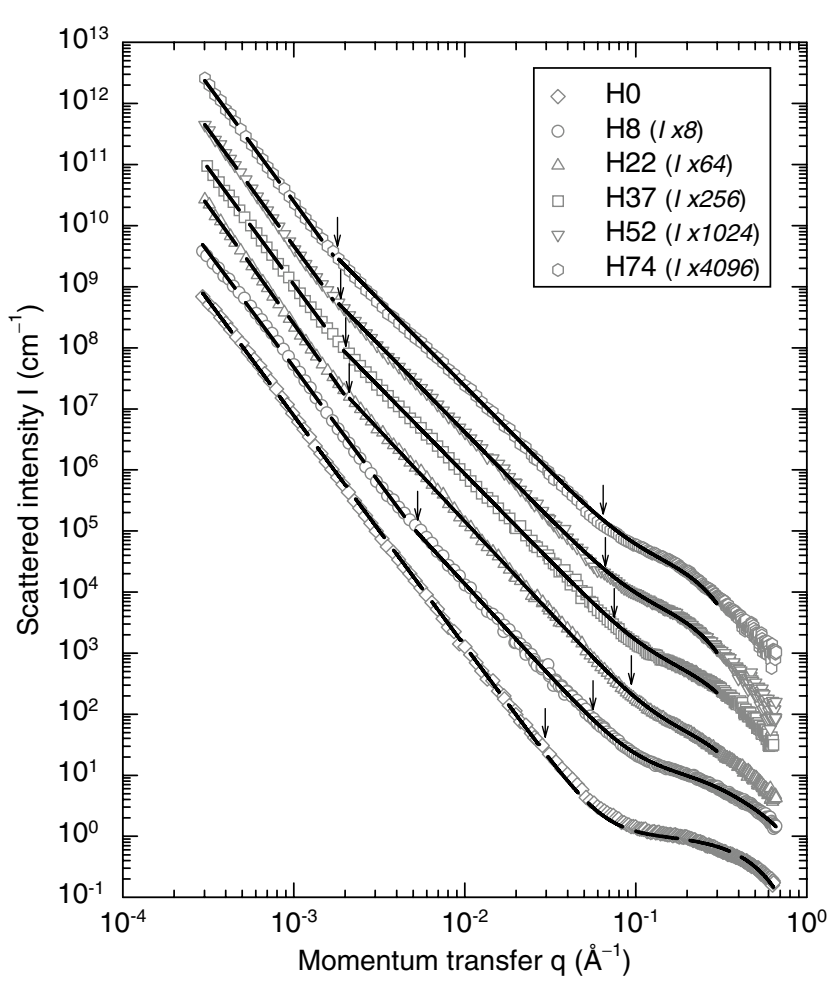

FIG. 1. SAXS curves. The full curves show the fit for the pore-fractal regime, Eq. (2) $\left[I_{0}=0\right.$ for $\left.\mathrm{H} 22-\mathrm{H} 74\right]$. The dashed curves show the fit for the surface-fractal regime [Eq. (1) for $\mathrm{H} 0 ; I(q) \propto q^{D_{\mathrm{s}}-6}$ for $\left.\mathrm{H} 8-\mathrm{H} 74\right]$. Arrows indicate the nominal end of the respective power-law regimes.

[17]; for a mass fractal, $D_{\mathrm{s}}=D_{\mathrm{m}}$ and $D_{\mathrm{p}}=3$, it reduces to $I(q) \propto q^{-D_{\mathrm{m}}}[18]$; and for a pore fractal, $D_{\mathrm{s}}=D_{\mathrm{p}}$ and $D_{\mathrm{m}}=3$, it reduces to $I(q) \propto q^{-D_{\mathrm{p}}}$ [15]. Sample H0 exhibits an extended power law, corresponding to a surface fractal with $D_{\mathrm{s}}=2.23$ at length scales $2.0 \times 10^{2} \AA\left(=L_{\mathrm{s}}\right) \leq 2 \pi / q \leq 2.1 \times 10^{4} \AA \quad[13,19]$. At shorter lengths, the fractal structure is masked by scattering from randomly distributed voids in the char (knee in the scattering curve). The two contributions are independent (additive intensities) and fitted with

$$
I(q)=\text { const } \times q^{D_{\mathrm{s}}-6}+I_{0} e^{-q^{2} L_{0}^{2} / 20} .
$$

The exponential is the Guinier approximation for scattering from a sphere of diameter $L_{0}$, yielding $L_{0}=10 \AA$. The activated samples, $\mathrm{H} 8-\mathrm{H} 74$, show the same surfacefractal structure above $4000 \AA$, but power laws $q^{-\alpha}$ with $\alpha \leq 3$, characteristic of mass or pore fractals, at shorter lengths. Since the oxidation process "drills" channels into the char [10-13] (confirmed by the adsorption data below), mass fractals are ruled out. The expression for the pore-fractal structure is

$$
I(q)=I_{\mathrm{p}}\left[1+\left(q L_{\min } / 2\right)^{-D_{\mathrm{p}}}\right] e^{-q^{2} L_{\min }^{2} / 20}+I_{0} e^{-q^{2} L_{0}^{2} / 20},
$$

valid for $q \geq 2 \pi / L_{\max }$, where $L_{\min }$ is the channel width and $L_{\max }$ is the network diameter (inner/outer cutoff of the fractal regime, respectively). Equation (2) represents the network as an "aggregate of spherical pores" (dimension $D_{\mathrm{p}}$, primary pore diameter $L_{\min }$ ), with structure factor given by the term in brackets $[16,18]$ and form factor $e^{-q^{2} L_{\min }^{2} / 20}$, using Babinet's principle. When networks touch, as they may, the overall network is fractal at scales below $L_{\max }$ and uniform at scales above $L_{\max }$. The random-void term exists in all samples, but is rapidly overtaken by the network term and is dropped, $I_{0}=0$, in $\mathrm{H} 22-\mathrm{H} 74$ (it can no longer be fitted with significance). The fits yield $D_{\mathrm{p}}=2.81-3.03, L_{\min }=15-20 \AA$, $L_{\max }=1.2 \times 10^{3}-3.4 \times 10^{3} \AA$ (Table I) [20]. The external surface undergoes no discernible change (constant $D_{\mathrm{s}}[\alpha \sim 3.8]$, masked at large $q$ by scattering from the internal surface $[\alpha \sim 2.9])$. In H8, the network is still small and $D_{\mathrm{p}}$ may be on the high side by an admixture from the power law $I(q) \propto q^{-3.77}$ (likewise in $\mathrm{H} 22$ ). The asymptotic fractal network is reached in H37, with $D_{\mathrm{p}}=2.85, \quad L_{\min }=16 \AA, \quad L_{\max }=3.2 \times 10^{3} \AA$, and little change upon further oxidation. The high $D_{\mathrm{p}}$ values mark the networks as nearly space filling. It is remarkable that $\mathrm{H} 22-\mathrm{H} 74$ can be fitted with just three parameters at $q>0.002 \AA^{-1}$ and span a scaling regime of $L_{\max } / L_{\min } \sim 200$, comparable to the most extended scaling regimes known [17(a),21].

From these results we can estimate the porosity, $\phi_{\text {SAXs }}$, of a block of solid invaded by the network. The ratio of $\left(L_{\max } / L_{\min }\right)^{D_{\mathrm{p}}} L_{\text {min }}^{3}$ (network volume) to $L_{\text {max }}^{3}$ (networkplus-solid volume) gives

$$
\phi_{\mathrm{SAXS}}=\left(L_{\max } / L_{\min }\right)^{D_{\mathrm{p}}-3}
$$

For a network that fills space, $D_{\mathrm{p}}=3$, but not uniformly [17(b),17(c)], (3) does not apply. In this case we consider $V(L)$, the volume of the set of all points with distance $\leq L / 2$ from the network, taken as a branched curve. The set equals the pore space if $L=L_{\min }$, and the space occupied by pores and solid if $L=L_{\max }$. Fractal scaling and a correction term for small $L$ lead to $V(L)=$ $V_{1}\left(L / L_{\min }\right)^{3-D_{\mathrm{p}}}+V_{2}$, where the constants $V_{1}$ and $V_{2}$ are fixed by the conditions $V\left(L_{\min }\right)=V_{\text {pore }}$ (pore volume) and $V^{\prime}\left(L_{\min }\right)=V_{\text {pore }} / L_{\min }$ (pore area) [22]. This yields

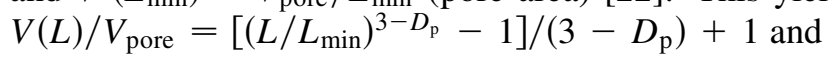

TABLE I. Fractal structure from SAXS and $\mathrm{N}_{2}$ adsorption. The $\phi_{\text {SAXS }}$ values for $\mathrm{H} 8$ and $\mathrm{H} 22$ are calculated from Eq. (4), those for H37-H74 from (3). The $V_{\mathrm{N}_{2}}$ and $\phi_{\mathrm{N}_{2}}$ values are for mass $m_{\text {solid }}=1.0 \mathrm{~g}$ and density $\rho_{\text {solid }}=2.0 \mathrm{~g} / \mathrm{cm}^{3}$ (amorphous carbon). Error bars include variations in different batches.

\begin{tabular}{ccccccc}
\hline \hline & H0 & H8 & H22 & H37 & H52 & H74 \\
\hline$D_{\mathrm{s}}( \pm 0.05)$ & 2.23 & 2.23 & 2.12 & 2.19 & 2.18 & 2.18 \\
$L_{\mathrm{s}}\left(10^{3} \AA\right)$ & 0.20 & 1.4 & 3.2 & 4.0 & 3.7 & 3.7 \\
$D_{\mathrm{p}}( \pm 0.05)$ & $\cdots$ & 3.03 & 2.99 & 2.85 & 2.85 & 2.81 \\
$L_{\min }(\AA)$ & $\cdots$ & 17 & 15 & 16 & 20 & 20 \\
$L_{\max }\left(10^{3} \AA\right)$ & $\cdots$ & 1.2 & 3.0 & 3.2 & 3.4 & 3.4 \\
$L_{0}(\AA)$ & 10 & 8 & $\cdots$ & $\cdots$ & $\cdots$ & $\cdots$ \\
$\phi_{\mathrm{SAXS}}( \pm 0.05)$ & $\cdots$ & 0.23 & 0.19 & 0.45 & 0.46 & 0.38 \\
$V_{\mathrm{N}_{2}}\left(\mathrm{~cm}^{3}\right)$ & 0 & 0.25 & 0.39 & 0.56 & 0.61 & 0.84 \\
$\phi_{\mathrm{N}_{2}}( \pm 0.05)$ & 0 & 0.33 & 0.44 & 0.53 & 0.55 & 0.63 \\
\hline \hline
\end{tabular}




$$
\begin{aligned}
\phi_{\mathrm{SAXS}} & =\frac{V\left(L_{\min }\right)}{V\left(L_{\max }\right)}=\left[\frac{\left(L_{\max } / L_{\min }\right)^{3-D_{\mathrm{p}}}-1}{3-D_{\mathrm{p}}}+1\right]^{-1} \\
& \sim\left[\ln \left(L_{\max } / L_{\min }\right)\right]^{-1}
\end{aligned}
$$

for $D_{\mathrm{p}}=3$ and $L_{\max } \gg L_{\min }$. The results are $\phi_{\mathrm{SAXS}}=$ 0.19-0.46 (Table I) [23].

We turn to the nitrogen adsorption isotherms, Fig. 2. The nearly flat high-pressure uptake and weak hysteresis document the narrow range of pore sizes requisite for a pore fractal. By assigning a monolayer of thickness $n^{-1 / 3}$ at $P / P_{0}=0.05$ ( $n$ : number density of liquid $\mathrm{N}_{2}$ ) [24] and a film of thickness $L_{\min } / 2$ at $P / P_{0}=0.99$ (full channel) to each of two opposite walls of a channel, we can relate the uptake to the channel width:

$$
N(0.99) / N(0.05)=(1 / 2) L_{\min } n^{1 / 3} .
$$

Were the samples mass fractals, the volume uptake at the two pressures would be $\phi_{\mathrm{SAXS}} L_{\max }^{3} L_{\min }^{-1} n^{-1 / 3}$ (monolayer on an aggregate with volume fraction $\phi_{\mathrm{SAXS}}$ occupied by mass) and $\left(1-\phi_{\mathrm{SAXS}}\right) L_{\max }^{3}$ (pore volume of the aggregate filled with capillary condensate), giving

$$
N(0.99) / N(0.05)=\left(\phi_{\mathrm{SAXS}}^{-1}-1\right) L_{\min } n^{1 / 3} .
$$

Estimates (5) and (6), evaluated with the SAXS data, predict uptake ratios of $1.9-2.6$ and $5-17$, respectively. Thus the experimental ratios, $N(0.99) / N(0.05)=1.2-1.9$ (Fig. 2), agree with the pore-fractal scenario within a factor of order 1, and disagree with the mass-fractal scenario by a factor of order 10 . The widths from (5), $L_{\min }=9-15 \AA$, agree with those from SAXS; they are on the low side because in narrow pores $N(0.05)$ overestimates the monolayer value [24]. The quantities $V_{\mathrm{N}_{2}}=$

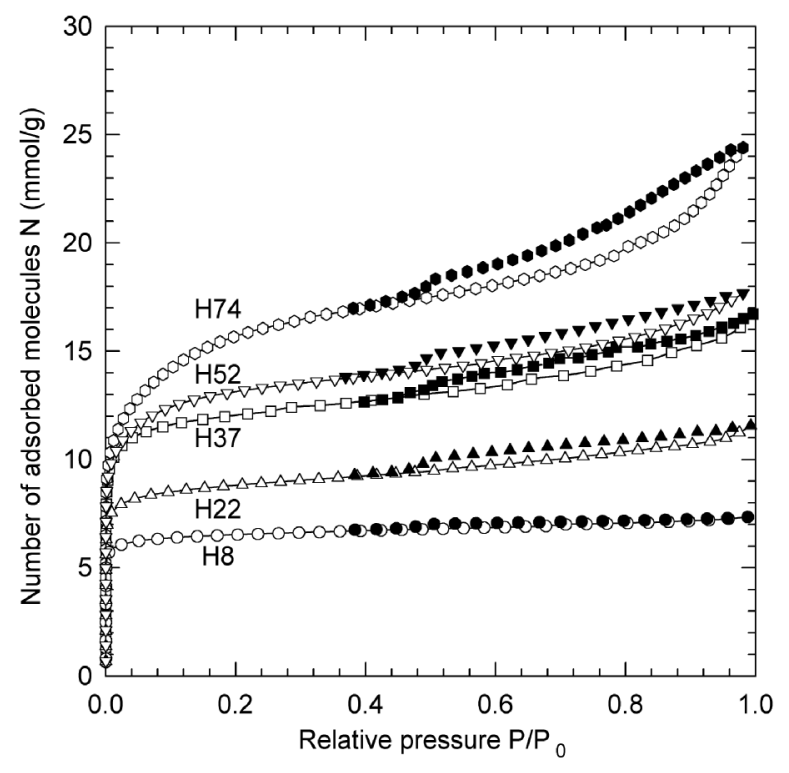

FIG. 2. $\mathrm{N}_{2}$ adsorption (open symbols) and desorption (full symbols) isotherms at $77 \mathrm{~K}$, with $P$ the vapor pressure and $P_{0}$ the coexistence pressure of bulk liquid and vapor. H0 adsorbs $\mathrm{N}_{2}$ negligibly on the scale of this graph.
$N(0.99) / n$ and $\phi_{\mathrm{N}_{2}}=V_{\mathrm{N}_{2}} /\left(V_{\mathrm{N}_{2}}+m_{\text {solid }} / \rho_{\text {solid }}\right)$ estimate the network's volume and porosity (Table I). As they include condensate in external pores [12] (isotherm rise in H37-H74), they are upper bounds. The agreement of $\phi_{\text {SAXS }}$ and $\phi_{\mathrm{N}_{2}}$ (mostly within error bars) and compliance with $\phi_{\mathrm{SAXS}} \leq \phi_{\mathrm{N}_{2}}$ shows that the high porosity, $\phi_{\mathrm{N}_{2}}=$ $0.33-0.63$, resides mostly in pores of width $15-20 \AA$.

Since most channels of a pore fractal are deep inside the solid, invisible from the outside, and fill the solid nonuniformly, one expects sparse entrances at the external surface. Scanning tunneling microscopy (STM, Fig. 3) confirms this. The channel width of $13 \pm 7 \AA$ for H37, sampled over 42 images, agrees with $L_{\min }=16 \AA$ from SAXS, sampled over the whole network. So the width at the entrance and in the solid is the same. Selected as an STM-imageable region, Fig. 3 shows little external roughness. Roughness consistent with $D_{\mathrm{s}}=2.19$ at large scales is seen in scanning electron micrographs [11].

What drives the formation of pore fractals in our samples? To answer this question, we return to $\mathrm{H} 0$, which has an easily accessible external surface $\left(D_{\mathrm{s}}=2.23\right)$, no open pores in which $\mathrm{N}_{2}$ would condense, and closed pores with mean diameter $L_{0}=10 \AA$. From $V_{\text {pore }}(\mathrm{H} 0)=$ $V_{\mathrm{N}_{2}}(\mathrm{H} 8)-0.08 m_{\text {solid }} / \rho_{\text {solid }}=0.21 \mathrm{~cm}^{3}$ we find the volume fraction of closed pores in $\mathrm{H} 0$ as 0.30 , which gives a mean distance of $12 \AA$ between centers of neighboring voids. Oxidation by $\mathrm{H}_{2} \mathrm{O}$ removes the weakest wall between the solid's exterior and a nearby closed pore, creating a channel through which new $\mathrm{H}_{2} \mathrm{O}$ removes the next weak wall, and so on. This carves out an invasion percolation cluster. A planar invasion percolation cluster has fractal dimension $1.90\left(=1+\frac{43}{48}\right)$ [25], and a stack of such clusters in registry has dimension 2.90. This is the model we propose for $\mathrm{H} 8-\mathrm{H} 74$, consistent with

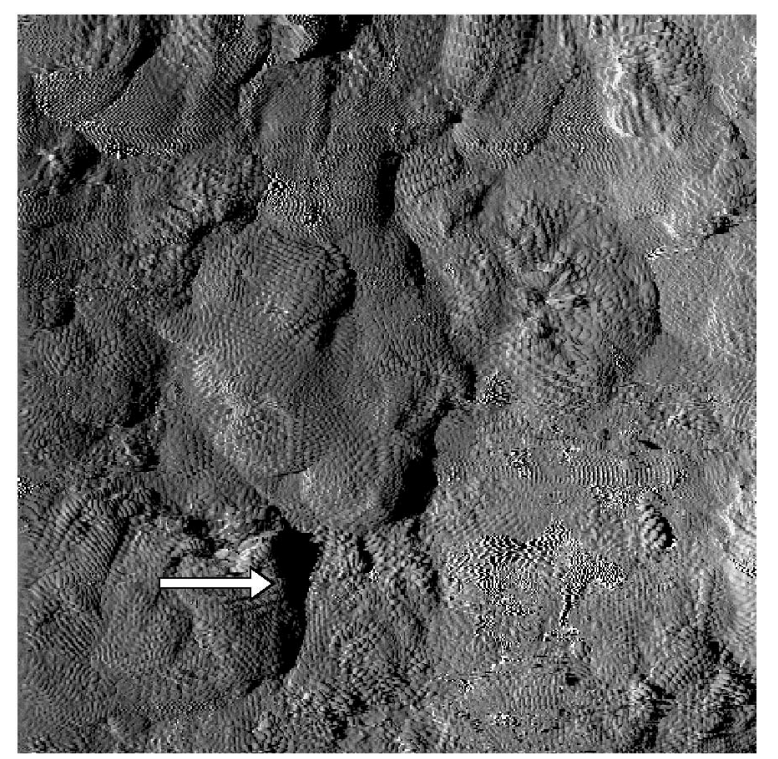

FIG. 3. STM image of sample H37 (320 $\times 320 \AA)$. Solid black areas are channel entrances. The width of the entrance marked with an arrow is $14 \AA$, at that location. 
$D_{\mathrm{p}}=2.9 \pm 0.1$, that $I_{\mathrm{p}}$ and $N(0.05)$ grow and $I_{0}$ drops with increasing burnoff, that pore mouths lie on parallel fault lines (running vertically in Fig. 3, left half), and that noninvaded regions, presumably pseudomorphs of fibrils of the biological precursor [26], are in registry transverse to the fault planes (Fig. 3, lower right quadrant). Without registry, the stack would be uniform above a few planes and scatter only at $q \sim 2 \pi /$ (spacing of planes). The model predicts that $L_{\min }$ remains constant and the width of the invaded zone, $L_{\max }$, grows with increasing burnoff, as observed. The growth stops when the accumulation of $\mathrm{H}_{2}$ deep inside the network slows the removal rate of weak walls down to the removal rate of strong walls at the external surface: the invasion front then advances in unison with the receding external surface, and $D_{\mathrm{p}}$ remains constant because strong walls stay inert until they become part of the external surface (H37-H74).

We expect pore fractals similar to the ones established here to form under a variety of conditions. However, for oxidation of $\mathrm{HO}$ with $\mathrm{CO}_{2}, \mathrm{CO}_{2} / \mathrm{H}_{2} \mathrm{O}$ mixtures and at higher temperatures, we find broad pore-size distributions [12] and surface fractals [13]. Most likely, the conditions for "pore aggregation" to yield pore fractals will be as complex as the conditions are for particle aggregation to yield mass fractals.

This work was supported by the Petroleum Research Fund, Grant No. 30602-AC9,5 (P.P.); the Department of Energy, Contracts No. W-7405-ENG-36 (P.P.) and No. DE-AC04-00A185000 (T.P. R.); and the Ceramic and Non-Metallic Materials Program at AFOSR (W.P.H.).

*Present address: Laboratoire de Spectrométrie Physique, CNRS, Université Joseph Fourier, BP 87, F-38402 SaintMartin d'Hères Cedex, France.

†Present address: Corporate Research, Corning Inc., SP-FR-03, Corning, NY 14831.

${ }^{\dagger}$ Deceased.

[1] D. Avnir, D. Farin, and P. Pfeifer, Nature (London) 308, 261 (1984).

[2] S. Havlin and D. Ben-Avraham, Adv. Phys. 36, 695 (1987); P. Pfeifer, in Preparative Chemistry Using Supported Reagents, edited by P. Laszlo (Academic Press, New York, 1987), p. 13; P. M. Adler and J.-F. Thovert, Transp. Porous Media 13, 41 (1993); M. A. Knackstedt et al., Phys. Rev. Lett. 75, 653 (1995); M. Appel et al., Europhys. Lett. 34, 483 (1996); J. S. Andrade et al., Phys. Rev. E 55, 772 (1997); Z. Gao and Z. R. Yang, Phys. Rev. E 60, 2741 (1999).

[3] O. Penrose, J. Stat. Phys. 45, 69 (1986); S. Wu and Z. R. Yang, J. Phys. A 28, 6161 (1995); R. Burioni et al., Phys. Rev. Lett. 85, 1496 (2000); G.-P. Zheng and M. Li, Phys. Rev. E 62, 6253 (2000).

[4] A. J. Katz and A. H. Thompson, Phys. Rev. Lett. 54, 1325 (1985); C.E. Krohn and A.H. Thompson, Phys. Rev. B 33, 6366 (1986); A. H. Thompson, Annu. Rev. Earth Planet
Sci. 19, 237 (1991); S. Roy and S. Trafdar, Phys. Rev. B 55, 8038 (1997).

[5] P. M. Adler, Porous Media (Butterworth-Heinemann, Boston, 1992); M. Sahimi, Rev. Mod. Phys. 65, 1393 (1993).

[6] A. C. Dillon et al., Nature (London) 386, 377 (1997); G. Stan et al., Phys. Rev. B 62, 2173 (2000); S. Talapatra et al., Phys. Rev. Lett. 85, 138 (2000).

[7] A. M. Rouhi, Chem. Eng. News 78, No. 34, 40 (2000).

[8] U. Even et al., Phys. Rev. Lett. 52, 2164 (1984); W. D. Dozier et al., Phys. Rev. Lett. 56, 197 (1986).

[9] C. L. Yang et al., J. Phys. Chem. 89, 3442 (1985); J. N. Roberts, Phys. Rev. Lett. 56, 2111 (1986); A. J. Katz and A. H. Thompson, Phys. Rev. Lett. 56, 2112 (1986); P.-Z. Wong et al., Phys. Rev. Lett. 57, 637 (1986); D. W. Schaefer et al., Phys. Rev. Lett. 58, 284 (1987); U. Even et al., Phys. Rev. Lett. 58, 285 (1987); J. Prasad and R. Kopelman, Phys. Rev. Lett. 59, 2103 (1987); A. Höhr et al., Phys. Rev. B 38, 1462 (1988); J. M. Drake and J. Klafter, Phys. Today 43, No. 6, 46 (1990).

[10] F. Derbyshire et al., in Porosity in Carbons: Characterization and Applications, edited by J. W. Patrick (Edward Arnold, London, 1995), p. 227.

[11] M. Iley et al., Carbon 11, 633 (1973).

[12] F. Rodríguez-Reinoso et al., Carbon 33, 15 (1995).

[13] F. Ehrburger-Dolle et al., in Fractals and Chaos in Chemical Engineering, edited by M. Giona and G. Biardi (World Scientific, Singapore, 1997), p. 27.

[14] T. P. Rieker and P. F. Hubbard, Rev. Sci. Instrum. 69, 3504 (1998).

[15] P. Pfeifer, in Multiple Scattering of Waves in Random Media and Random Rough Surfaces, edited by V. K. Varadan and V. V. Varadan (Pennsylvania State University, University Park, 1987), p. 45.

[16] R. Winter et al., Phys. Rev. E 60, 7354 (1999).

[17] (a) H. D. Bale and P. W. Schmidt, Phys. Rev. Lett. 53, 596 (1984); (b) P. Pfeifer and P. W. Schmidt, Phys. Rev. Lett. 60, 1345 (1988); (c) P. Pfeifer, D. Avnir, and D. Farin, NATO ASI, Ser. B 258, 215 (1991).

[18] T. Freltoft, J. K. Kjems, and S. K. Sinha, Phys. Rev. B 33, 269 (1986); J. Teixeira, J. Appl. Cryst. 21, 781 (1988).

[19] Porod's law for a sphere with radius $R$ and electron density $\rho_{\mathrm{e}}, I(q)=\left(6 \pi \rho_{\mathrm{e}}^{2} r_{\mathrm{e}}^{2} / R\right) q^{-4}\left(r_{\mathrm{e}}\right.$ : classical electron radius $)$, fitted to the data gives a particle or hole size of $R=$ 5-13 $\mu \mathrm{m}$, consistent with the low- $q$ end of the data and the presence of $5 \mu \mathrm{m} \times 20 \mu \mathrm{m}$ cracks [11].

[20] The scattering law $I(q) \propto q^{-3}$ is discussed in [17(b),17(c)].

[21] A. P. Radlinksi et al., Phys. Rev. Lett. 82, 3078 (1999); D. Broseta et al., Phys. Rev. Lett. 86, 5313 (2001).

[22] P. Pfeifer et al., Proc. R. Soc. London A 423, 169 (1989).

[23] Our estimates do not include the factor $a N_{\mathrm{i}} / N_{\mathrm{b}}$ that enters if only $N_{\mathrm{i}}$ of $N_{\mathrm{b}}$ blocks of the solid are invaded and the aspect ratio of the channel cross section is $a>1$.

[24] C. Lastoskie et al., J. Phys. Chem. 97, 4786 (1993).

[25] J. Feder, Fractals (Plenum Press, New York, 1988); Y. Zhang, Commun. Math. Phys. 167, 237 (1995).

[26] While carbonization often proceeds via a liquid-crystal state [R. H. Hurt and Z.-Y. Chen, Phys. Today 53, No. 3, 39 (2000)], our samples form through an all-solid-state route, preserving fibrillar structures of the precursor [11]. 